Treatment of bone metastasis is a major challenge for current and future orthopedic and oncology specialists. For the treatment of single metastases, there is a continuing search for effective local treatments that do not affect the whole patient. The current report describes the case of single breast cancer metastasis to the pelvic bone in a 50-year-old woman treated surgically by bone osteotomy combined with local doxorubicin application. The presence of cancer cells was confirmed by histopathological examination. After 6 months, the same defect was operated on again and a successful therapeutic result was confirmed by negative tumor pathology tests. The bone defect caused by osteotomy was reconstructed with allogenic bone grafts that healed completely over 14 months. To the best of our knowledge, this report is the first to combine the removal bone metastasis with direct local cytostatic drug administration without the use of a specific carrier, followed by successful bone reconstruction 6 months later using bone allografts. Thus, single bone metastasis may be successfully treated with resection and local cytostatic administration, enabling bone reconstruction in the postoperative period. The potential benefits of such treatments must be evaluated by considering the potential risks involved, including necrosis of surrounding soft tissues and general reactions to the chemotherapy. No data has been available until now regarding the risk of cancer disease generalization being influenced by such treatments, but patient quality of life can be improved significantly by the successful removal of a solitary bone metastasis. The present case report supports the continuation of research on improving local cytostatic drug administration during the treatment of bone metastasis.

Key words: bone metastasis, breast cancer, local chemotherapy, doxorubicin.

Contemp Oncol (Pozn) 2017; 21 (4): 306-310 DOI: https://doi.org/10.5114/wo.2017.72402

\section{Solitary breast cancer metastasis to pelvic bone treated with a unique method of surgery combined with local doxorubicin administration}

\author{
Andrzej Bohatyrewicz, Maciej Karaczun, Daniel Kotrych, Paweł Ziętek, \\ Łukasz Kołodziej, Alina Jurewicz
}

Department of Orthopaedics, Traumatology and Orthopaedic Oncology, Pomeranian Medical University of Szczecin, Pomeranian Medical AcademyOncology Therapy and Research Center, Szczecin, Poland

\section{Introduction}

Metastatic bone disease is far more common than primary bone tumors. The systemic treatment of bone metastasis includes chemotherapy, radiotherapy, antiresorptive drugs and pain management with analgesics. Local therapy includes radiation and surgical stabilization of impending and pathological fractures, or the implantation of tumor prostheses in selected cases.

Local cytostatic treatment of primary malignant tumors has been investigated in certain studies, with varying results; although positive effects from intravesical administration of cytotoxic agents in bladder cancer have been reported [1], there have been discouraging reports of extensive toxicity associated with intraarterial cytostatic drug administration for locally advanced breast cancer [2]. Additionally, novel perspectives have been provided by researchers investigating the technical possibility of adding antiblastic drugs to bone cement and using this to fill the metastatic bone defects following curettage [3-7]. To the best of our knowledge, the direct application of cytostatic agents into the bone cavity following the removal of metastatic tissue has not yet been reported.

The aim of our study is to describe a case of single breast cancer metastasis to the pelvic bone in female patient treated surgically and combined with local doxorubicin application. Such treatment enabled later successful bone reconstruction with allogenic bone grafts.

\section{Case report}

A single breast amputation was performed on a 45-year-old woman with unifocal, unicentric breast cancer without affection of lymph nodes breast cancer and without any signs of disease generalization. Postoperative pathology revealed a poorly differentiated, grade 3 tumor with highly proliferative cells. Following tumor parameters were additionally determined estrogen receptors were positive, Herceptin test was negative. No data exist about estimation of progesterone receptors and Ki-67 antigen. The patient received six courses of doxorubicin and cyclophosphamide chemotherapy, followed by local radiotherapy. No hormonal treatment was used. At 12 months after the breast amputation, radical hysterectomy and adnexectomy were performed. At 2 and 3 years after breast amputation, tumor markers were within the reference range for healthy individuals.

At 4 years after mastectomy, the patient began to complain of pain in the right ilium. Anteroposterior pelvic X-ray revealed an osteolytic lesion in the 
right iliac bone localized above the acetabular roof. Carcinoma Antigen 15-3 and Carcinoembryonic antigen tumor markers in the blood were increased significantly. Bone scans confirmed that the lesion was active and probably metastatic; however, similar abnormalities were not detected in the remaining skeleton. The right ilium was irradiated with 20 Gy radiotherapy. After 3 months, computed tomography (CT) was performed and a bone scan of the pelvis repeated. CT revealed a closed osteolytic lesion in the right iliac bone, $\sim 15 \mathrm{~cm}^{3}$ in volume without cortex penetration (Fig. 1). The bone scans demonstrated increased osteoblastic activity with high metastatic potential despite the radiotherapy.

Based on the clinical state of the patient and the results of imaging, the interdisciplinary team, consisting of an orthopedic surgeon, pathomorphology specialist and oncology specialist, devised the therapeutic strategy, presented it to the patient and obtained written consent. The therapy was based on an open biopsy of the lesion, with the expectation of tumor-positive intraoperative pathology and planned administration of doxorubicin into the bone.

The patient underwent surgery in the supine position; the highest and most anterior possible site of bone pene-

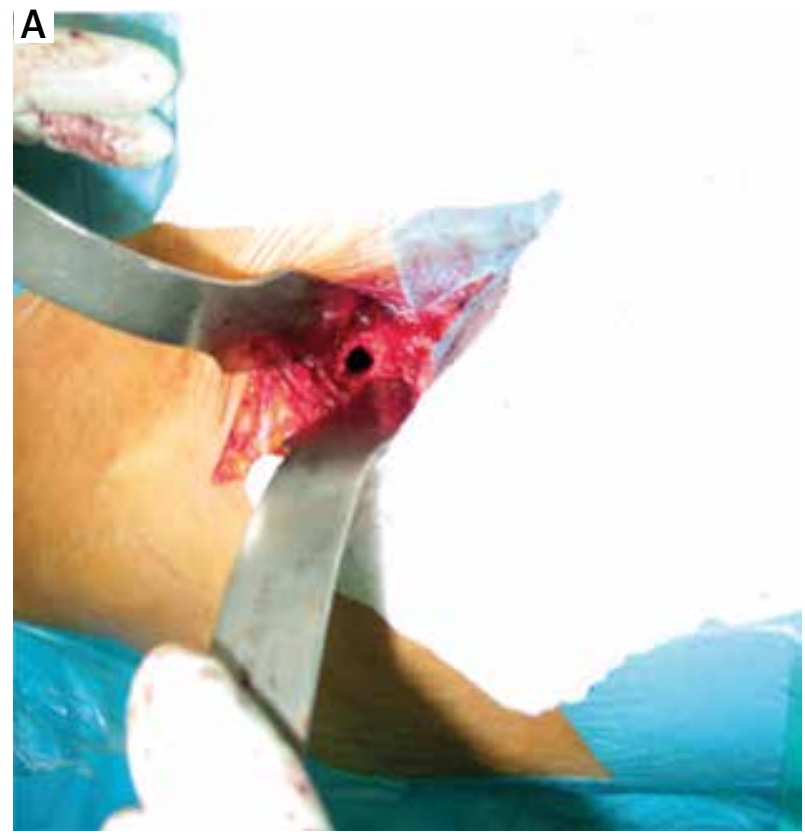

Fig. 2. Intraoperative pictures demonstrating (A) surgical exposure of the pelvic bone, (B) doxorubicin administration to the pelvic lesion and $(\mathbf{C})$ closure of the osteotomy site following doxorubicin application

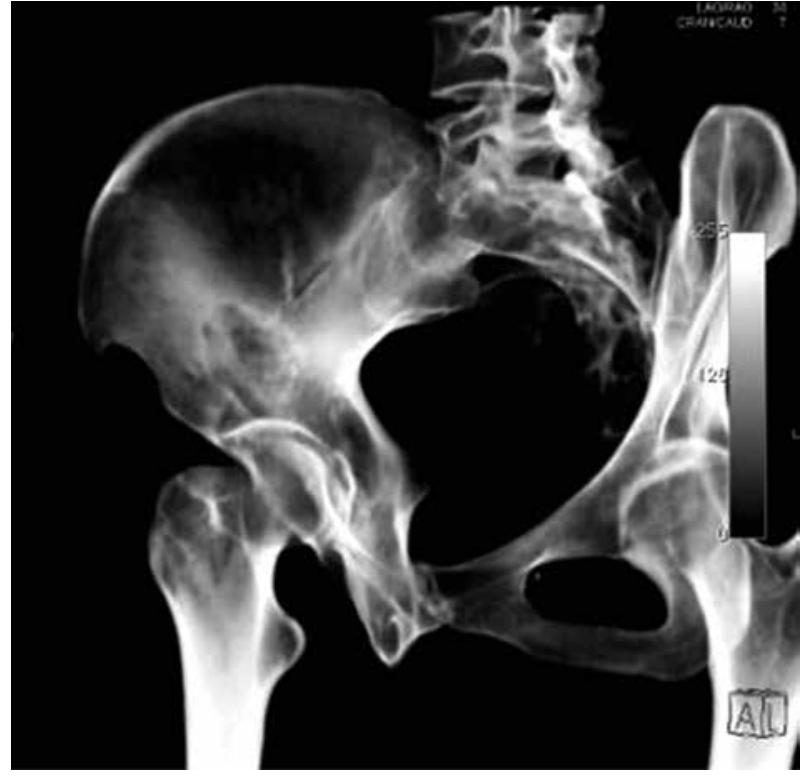

Fig. 1. Computed tomography scan presenting the supraacetabular location of bone metastasis prior to surgery
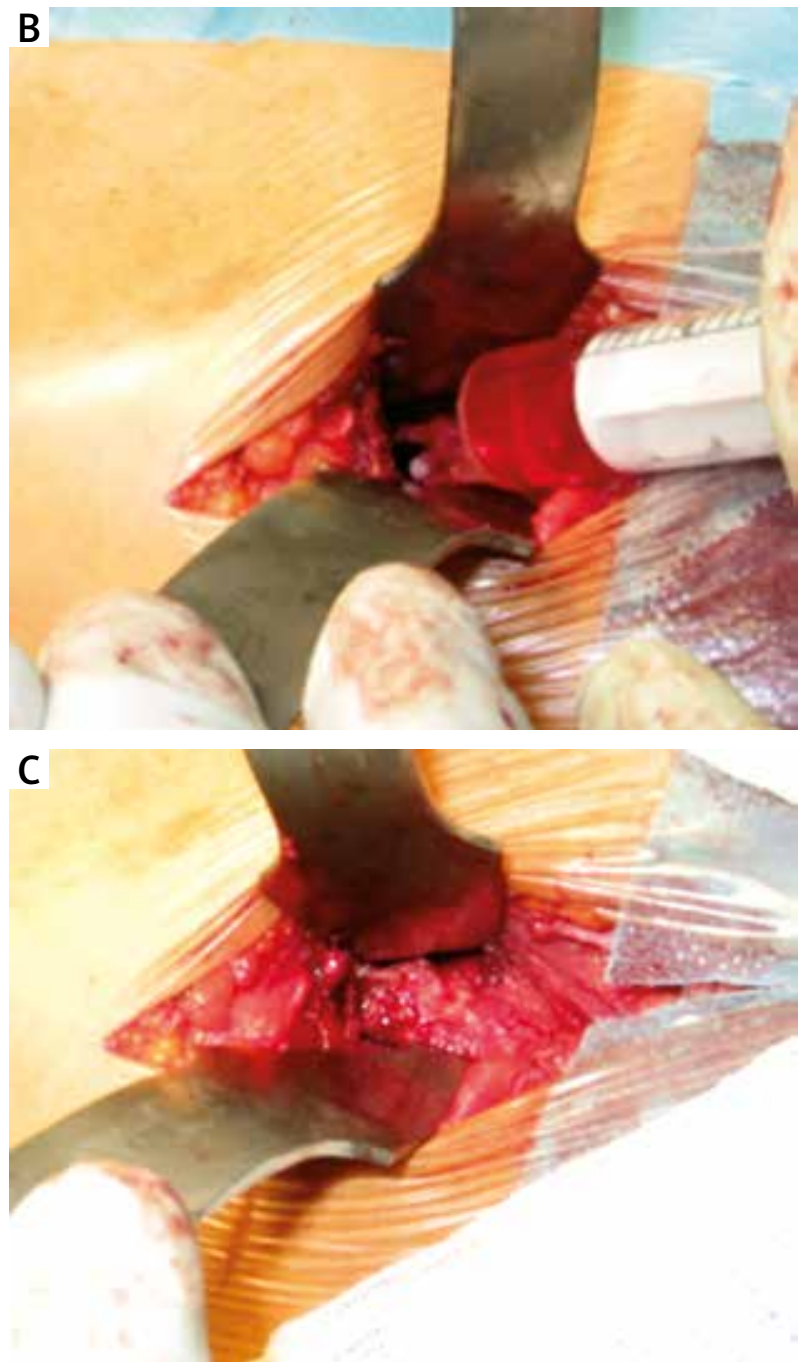


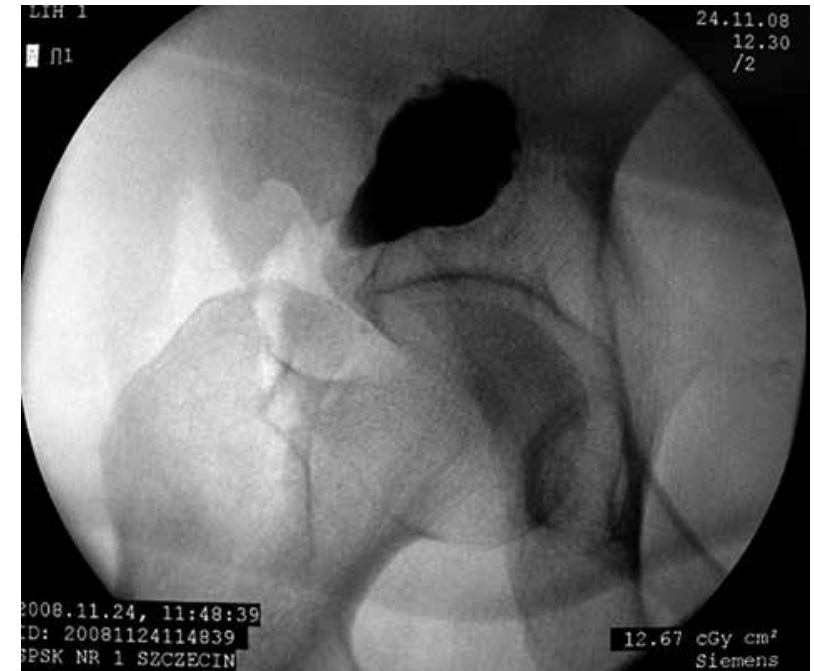

Fig. 3. Anteroposterior intraoperative $\mathrm{X}$-ray showing the location of the metastasis. The contrast material was confined to the cavity, indicating that it was sealed
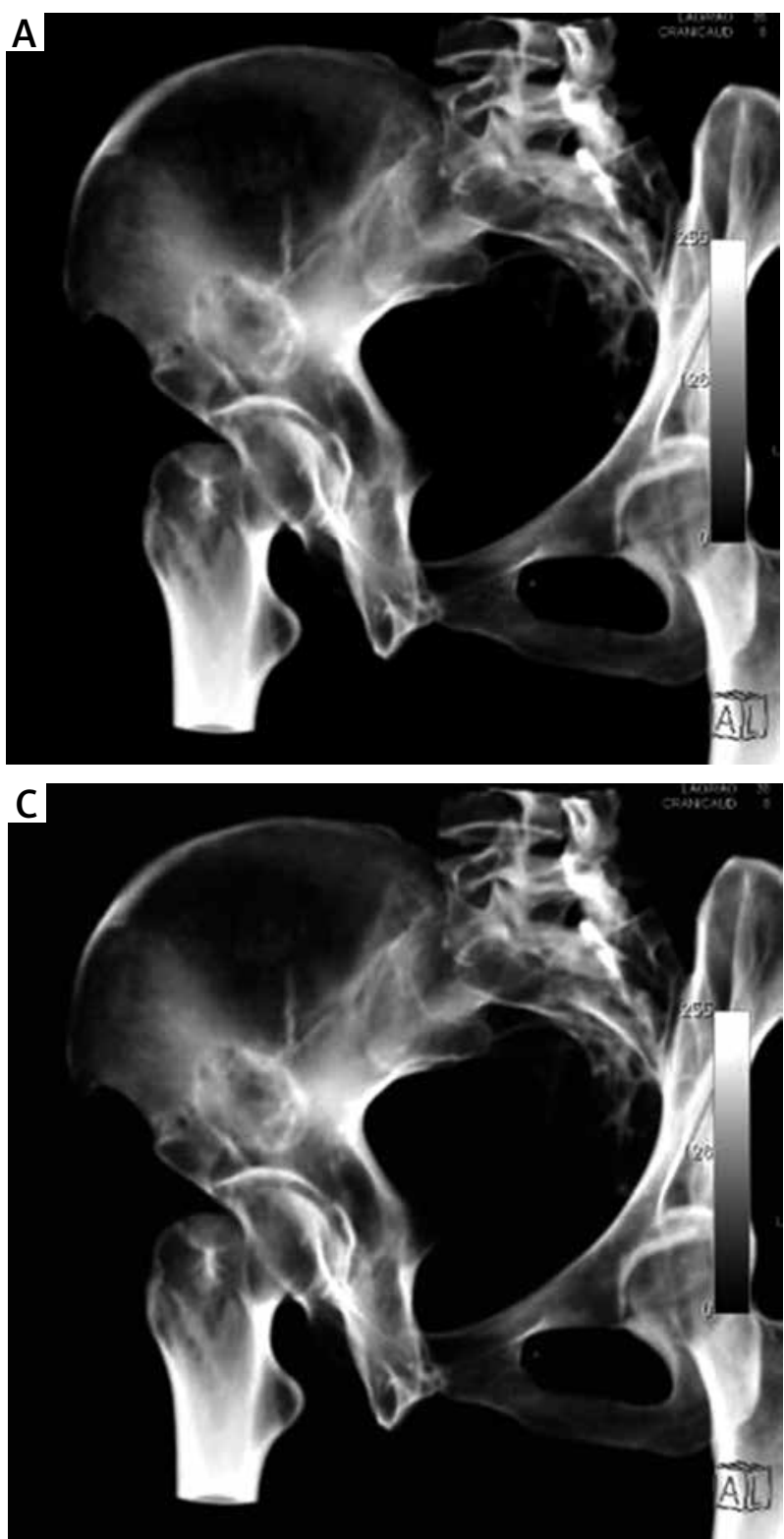

tration in the supraacetabular region was chosen, allowing the drug to be administered precisely without contact with surrounding tissues or the possibility of leakage from the administration site (Fig. 2A). Fluoroscopy was used to identify the operated area [7]. The diameter of the osteotomy site was $1 \mathrm{~cm}$ and the roof of the bone was preserved for closure of the resected area. The intracystic material was removed. Pathomorphology analysis confirmed the presence of tumor cells and $5 \mathrm{mg}$ doxorubicin liquid (2.5 $\mathrm{ml}$; suspended in a solution of sodium chloride and hydrochloric acid) was applied to the defect (Fig. 2B). The cavity was closed with the piece of bone cortex obtained during osteotomy (Fig. 2C). The tightness of the cavity was checked intraoperatively using contrast liquid (Fig. 3). Drainage was applied.

Postoperative CT was performed during the hospitalization period (Fig. 4A). During the postoperative period, no local or general complications occurred. The sutures were removed and the patient was discharged 10 days af-

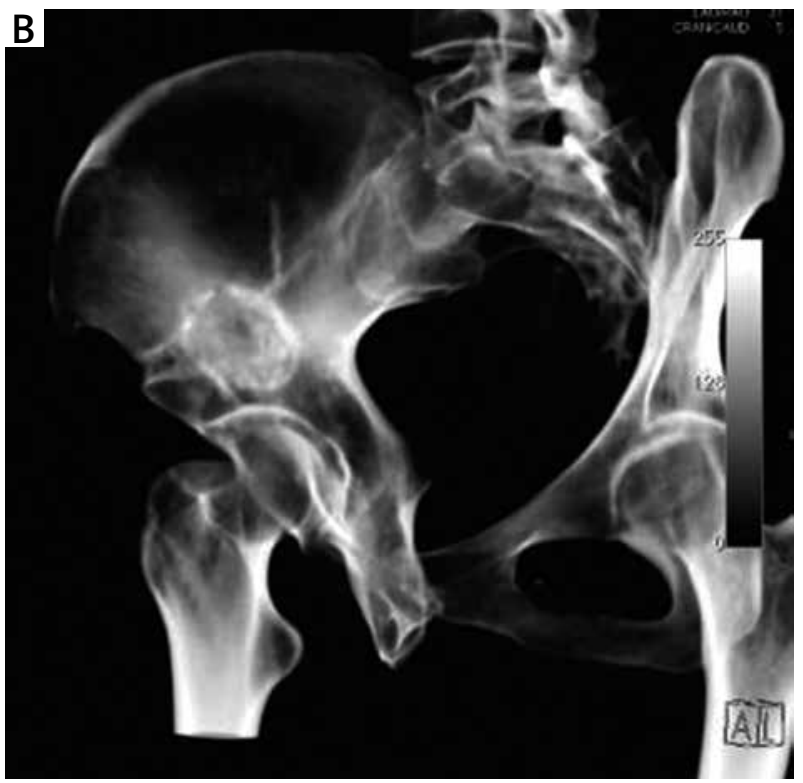

Fig. 4. Computed tomography scans showing (A) the pelvic bone following metastasis removal and filling of the cavity with doxorubicin, (B) the bone cavity 4 months after reconstruction using bone allografts and $(\mathbf{C})$ the completely healed bone allografts 14 months after implantation 
ter the surgery. Tumor markers decreased to normal levels 3 months after the surgery.

During the following 6-month observation period, the patient had no signs of recurrence. No pain was reported, only mild discomfort within the first 6 weeks after the surgery, and her gait was normal. After 6 months, a second operation was performed on the same area of the bone and intraoperative pathological analysis for the presence of tumor cells was negative. The defect was reconstructed using bone allografts. The metastatic region was radiologically evaluated by $\mathrm{CT}$ at 4 and 14 months after the second operation, revealing complete healing of the bone allografts (Fig. 4B and C).

\section{Literature review}

Treatment of metastatic bone lesions has predominantly been based on complete excision, acrylic cement implantation, internal fixation, or arthroplasty of the bone $[3,8,9]$. Thus far, many researchers have tried to evaluate the effect of local chemotherapy and find markers for its use in the treatment of bone tumors. In studies using rats and dogs, and preliminary clinical trials in patients with osteosarcoma, Hernigou et al. [10, 11] performed local tumor resection and local chemotherapy using methotrexate (MTX) in methylpolymethacrylate (PMMA); this increased survival outcomes and reduced the risk of relapse. The authors observed certain side effects associated with local chemotherapy, including gingivitis, glossitis, pharyngitis, weight loss, vomiting, diarrhea and impaired wound healing, which was hypothesized to be a result of too high a dose of chemotherapeutic agents [10,11].

Maccauro et al. [5] conducted clinical trials in patients with bone metastases from primary breast cancer. The treatment involved metastasis resection and local chemotherapy with MTX, and the resected area of bone was filled with PMMA. The simultaneous use of MTX and PMMA did not damage the supporting properties of PMMA or the chemotherapeutic effect of MTX [5].

Similar conclusions were made by Greco et al. [6]. PMMA was combined with doxorubicin and cisplatinum individually, and did not alter chemotherapeutic effect of these two agents on tumor cells in vitro. Additionally, no defects in PMMA polymerization were observed [12].

Özben et al. [13] determined a safe dose of doxorubicin combination with PMMA, which did not affect the mechanical properties of PMMA (50 mg per $40 \mathrm{~g}$ of PMMA) [13]. Modern chemotherapy protocols have improved the prognosis in various types of cancer and, increasingly, there is discussion regarding the use of local chemotherapy to treat bone cancer $[3,14]$. Numerous studies have used PMMA to replace resected bone following surgery, with a good short-term results. However, there are no clear studies that have reported on the long-term mechanical strength of cement used to replace resected bone $[3,4,6$, 7, 15-17]. Furthermore, to the best of our knowledge, no studies have reported on the use of in vivo local chemotherapy followed by allogenic bone grafting thus far.

The major difficulty for local chemotherapy is the choice of antiblastic drug and evaluating its therapeutic dose [18].
The greatest local danger is necrosis of the surrounding soft tissues if the drug leaves site of bone resection. If the dose is too high, the general complications may lead to acute marrow aplasia and patient mortality [2]. The recommended doxorubicin dose in single monotherapy is 60-75 $\mathrm{mg} / \mathrm{m}^{2}$. On the basis of our present clinical experience, we suggest that administration of doxorubicin in low doses to the closed bone metastasis from primary breast cancer, with allogenic bone grafting as a secondary procedure, is a useful therapeutic strategy for the treatment of solitary bone lesions without pathological fracture or penetration into surrounding soft tissues. This type of treatment based on precise visualization of the metastasis and improved local cytostatic administration requires further development and improvement.

\section{The authors declare no conflict of interest.}

\section{References}

1. Nargund VH, Tanabslan CIK, Kabir MN. Management of non-muscle-invasive (superficial) bladder cancer. Semin Oncol 2012; 39: 559-72.

2. Twelves JC, Chaudary MA, Reidy J, Richards MA, Rubens RD. Toxicity of intra-arterial doxorubicin in local advanced breast cancer. Cancer Chemother Pharmacol 1990; 25: 459-462.

3. Healey JH, Shannon F, Boland P, DiResta GR. PMMA to stabilize bone and deliver antineoplastic and antiresorptive agents. Clin Orthop Relat Res 2003; 415 Suppl: 263-275.

4. Król R, Gusta A. Use of bone cements with added methotrexate for stabilization of pathologic fractures and filling of long bone defects in cases of neoplasm metastasis. Chir Narz Ruchu Ortop Pol 1998; 1: 73-80.

5. Maccauro G, Cittadini A, Casarci M, et al. Methotrexate-added acrylic cement: biological and physical properties. J Mater Sci Mater Med 2007; 18: 839-44.

6. Rosa M A, Maccauro G, Sgambato A, Ardito R, Falcone G, De Santis V, Muratori F. Acrylic cement added with antiblasts in the treatment of bone metastases. J Bone Joint Surg [Br] 2003; 85-B: 712-6.

7. Wang Z, Lu Y, Qiao D, Wen X, Zhao H, Yao Y. Diagnostic and prognostic validity of serum bone turnover markers in bone metastatic non-small cell lung cancer patients. J Bone Oncol 2015; 4: 85-91.

8. McDonald R, Chow E, Rowbottom L, et al. Quality of life after palliative radiotherapy in bone metastases: A literature review. J Bone Oncol 2014; 18: 24-31.

9. Kudawara I1, Aoki Y, Ueda T, et al. Neoadjuvant and adjuvant chemotherapy with high-dose ifosfamide, doxorubicin, cisplatin and high-dose methotrexate in non-metastatic osteosarcoma of the extremities: a phase II trial in Japan. J Chemother 2013; 25: 41-8.

10. Hernigou P, Brun B, Astier A, Goutallier D, le Bourgeois JP. Diffusion of methotrexate from surgical acrylic cement. Cancer Treat Res 1993; 62: 231-3.

11. Hernigou P, Thiéry JP, Benoit J, Voisin MC, Leroux P, Hagege G, Delepine G, Goutallier D. Methotrexate diffusion from acrylic cement. Local chemotherapy for bone tumours. J Bone Joint Surg Br 1989; 71: 804-11.

12. Greco F, de Palma L, Specchia N, Jacobelli S, Gaggini C. Polymethylmethacrylate-antiblastic drug compounds: an in vitro study assessing the cytotoxic effect in cancer cell lines - a new method for local chemotherapy of bone metastasis. Orthopedics 1992; 15: 189-94.

13. Özben H, Eralp L, Baysal G, Cort A, Sarkalkan N, Özben T. Cisplatin loaded PMMA: mechanical properties, surface analysis and effects on Saos-2 cell culture. Acta Orthop Traumatol Turc 2013; 47: 184-92. 
14. Paioli A, Luksch R, Fagioli F, et al. Chemotherapy-related toxicity in patients with non-metastatic Ewing sarcoma: influence of sex and age. J Chemother 2014; 26: 49-56.

15. Król R, Radomski S. Fixation of pathological fractures and impending long bone fractures in the course of neoplastic disease with the use of polimethylmethacrylate with added methotrexate. Ortop Traumatol Rehabil. 2003:5:319-326.

16. Lin WH, Lan TY, Chen Ch Y, Wu K, Yang RS. Similar local control between phenol and ethanol-treated giant cell tumors of bone. Clin Orthop Relat Res 2011; 469: 3200-8.

17. Wang HM, Crank S, Oliver G, Galasko CSB. The effect of methotrexate-loaded bone cement on local destruction by the VX2 tumor. J Bone Joint Surg [Br] 1996; 78-B: 14-7.

18. Lesniak MS, Upadhyay U, Goodwin R, Tyler B, Brem H. Local deliv ery of doxorubicin for the treatment of malignant brain tumors in rats. Anticancer Res 2005; 25: 2825-31.

\section{Address for correspondence}

\section{Maciej Karaczun}

Department of Orthopaedics,

Traumatology and Orthopaedic Oncology

Pomeranian Medical University of Szczecin,

Unii Lubelskiej 1

71-252 Szczecin, Poland

e-mail: karaczun@orthomail.com

Submitted: 30.08 .2017

Accepted: 22.09.2017 